CLINICAL PRACTICE

\title{
Acute intermittent porphyria presenting as progressive muscular atrophy in a young black man
}

\author{
C H Albertyn, M Sonderup, A Bryer, A Corrigall, P Meissner, J M Heckmann
}

Christine Albertyn is a neurology registrar in the Division of Neurology, Department of Medicine, Groote Schuur Hospital and University of Cape Town (UCT), South Africa. Mark Sonderup is a senior specialist in the Department of Medicine, Division of Hepatology and Lennox Eales Porphyria Laboratories, Groote Schuur Hospital and UCT. Alan Bryer is an associate professor and head of the Division of Neurology, Department of Medicine, Groote Schuur Hospital and UCT. Anne Corrigall is chief research officer in the Porphyria Laboratories, Department of Medicine, UCT. Peter Meissner is professor and head of the Division of Medical Biochemistry in the Department of Clinical Laboratory Sciences, a member of the Institute of Infectious Diseases and Molecular Medicine, Faculty of Health Sciences, and director of the Porphyria Laboratories, Department of Medicine, UCT. Jeannine Heckmann is an associate professor of Neurology in the Department of Medicine, UCT.

Corresponding author: C H Albertyn (christine.albertyn@uct.ac.za)

Acute intermittent porphyria, the most common porphyria affecting the nervous system, typically presents with neurovisceral crises followed by a motor neuropathy. We describe a 23-year-old black South African man presenting with a progressive stuttering, lower motor neuron syndrome developing over months. He had not experienced pain or neuropsychiatric symptoms. One year after symptom onset he was bed-bound with a flaccid quadriparesis. There was marked amyotrophy, but without fasciculations. Sensation was intact apart from a hypo-aesthetic patch over the thigh. Electrophysiological investigations showed an active motor axonopathy. Urinary porphyrins, $\delta$-aminolaevulinic acid and porphobilinogen were elevated. Mutation analysis revealed the $c 445 C>T$ (R149X) mutation in the porphobilinogen deaminase gene. The patient responded dramatically to haem arginate and could walk with assistance 2 weeks later. We identified the first molecularly confirmed acute intermittent porphyria in a black South African. The clinical presentation mimicked a progressive lower motor neuron syndrome.

S Afr Med J 2014;104(4):283-285. DOI:10.7196/SAMJ.7785



Acute intermittent porphyria (AIP), the most common porphyria affecting the nervous system, typically presents with neurovisceral crises followed by a motor neuropathy. We describe an unusual presentation in a young man with molecularly confirmed AIP.

\section{Case presentation}

A 23-year-old university student was referred to Groote Schuur Hospital, Cape Town, South Africa, with suspected motor neuron disease. Over the preceding year he had noticed insidious progressive weakness of both upper limbs. Initially, the weakness affected grip strength bilaterally, but within 9 months progressed to also involve proximal arm function. Ten months after onset, he developed profound leg weakness over a period of days, rendering him unable to walk without support. He had no bowel or bladder sphincter involvement and no bulbar symptoms, but complained of a weak cough. He had not experienced any neuropsychiatric symptoms or abdominal pain.

Examination showed a thin and wasted man with marked amyotrophy involving all four limbs, both proximally and distally (Fig. 1). He was bed-bound, and unable to turn in bed, feed or dress himself. He had mild symmetrical facial weakness and a weak cough with a reduced vital capacity ( $<80 \%$ of expected). He had truncal weakness and a flaccid quadriparesis, but no fasciculations were noted: upper limb strength Medical Research Council (MRC) grade $1 / 5$ proximal, $2 / 5$ distal; lower limb strength $2 / 5$ proximal, $3 / 5$ distal. He was areflexic in the legs with either hyporeflexia (biceps and supinator reflexes) or areflexia (triceps reflexes) in the arms. Findings on sensory examination were normal apart from a patch of reduced sensibility for pinprick and touch over the left anteromedial thigh region.
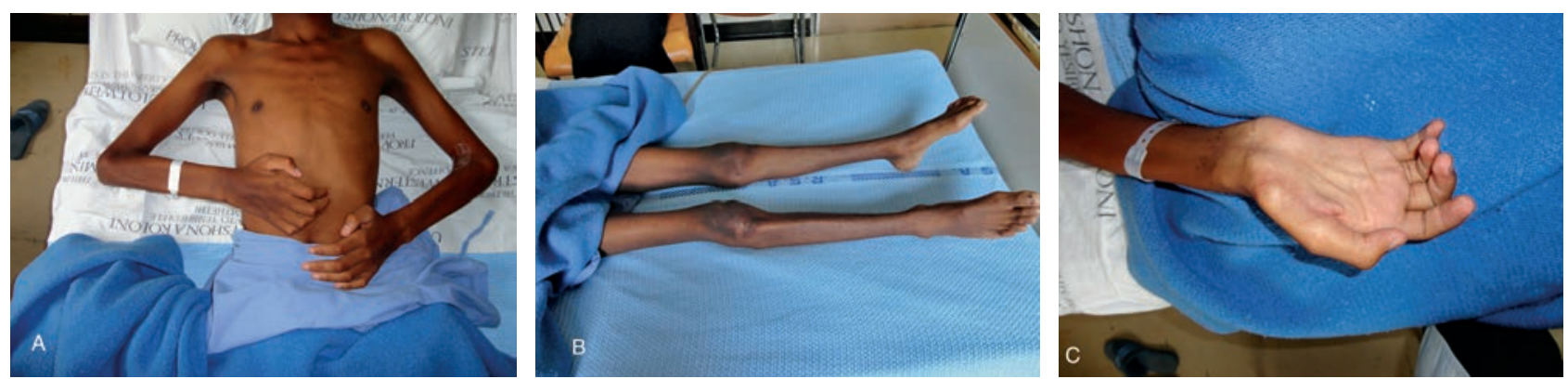

Fig. 1. (A) Upper limbs showing marked wasting of both proximal and distal muscles with flattening of forearms in the anteroposterior plane. (B) Lower limbs showing marked wasting of proximal and distal muscles with bilateral foot drop. (C) Dorsal aspect of right hand showing wasting of thenar and hypothenar muscles, and ulnar clawing. 
The following tests were either normal or negative: full blood count, renal function and electrolytes, liver profile, erythrocyte sedimentation rate, fasting glucose, $\gamma$-globulin electrophoresis, serum lead levels, HIV and syphilis serology. The cerebrospinal fluid was acellular with normal chemistry. Nerve conduction studies demonstrated a motor axonopathy in all four limbs with normal sensory responses. Electromyography studies of proximal and distal limb muscles showed denervation with fibrillation potentials in keeping with an active neurogenic process.

This unusual and severe motor neuropathy, but without fasciculations and a with single patch of numbness, prompted a porphyria screen. It was strongly positive with a $\delta$-aminolaevulinic acid (ALA) level of $678 \mu \mathrm{mol} / 10 \mathrm{mmol}$ creatinine (upper limit of reference range $\leq 45$ ) and a porphobilinogen (PBG) level of $1477 \mu \mathrm{mol} / 10 \mathrm{mmol}$ creatinine (upper limit of reference range s16). Plasma porphyrin fluoroscanning demonstrated an emission peak at a wavelength of $619 \mathrm{~nm}$ (excitation wavelength $405 \mathrm{~nm}$ ). This pattern, together with urinary ALA and PBG characterisation, was compatible with a diagnosis of AIP. His urinary porphyrin excretion profile showed greatly elevated porphyrin concentrations (10- to 13-fold) with a preponderance of uroporphyrin, typical of acute porphyria. The diagnosis of AIP was confirmed by identifying the R149X mutation, $c 445 C>T$ in exon 9 by sequencing the PBG deaminase gene (also known as hydroxymethylbilane synthase).

On additional questioning it became evident that 4 months after symptom onset, he had experienced paraesthesiae over both thighs and buttocks. This resolved partially over days with residual numbness over his left anterior thigh. Also, over the preceding 3 years he had had intermittent episodes of constipation, at times even requiring hospitalisation for laxative treatment.

\section{Clinical course}

The patient was treated with standard dosage intravenous haem arginate (Normosang), a formulation of haematin, for 6 days. He started improving within 3 days of treatment initiation and was able to turn in bed and hold his mobile phone to his ear. After 2 weeks he was able to stand and mobilise with assistance; both shoulder abduction and hip flexion had improved by at least one grade (shoulder $4 / 5$; hip $\geq 3 / 5$ ). The distal power remained unchanged.

Over the following 6 months, our patient re-presented twice with relapses: on one occasion characterised by abdominal pain and constipation; and on the other with only worsening hand weakness and constipation. Further courses of intravenous haem arginate were administered with resolution of abdominal pain and improvement in distal arm strength. He still has weak intrinsic hand muscles (MRC 2/5), but lives independently.

\section{Discussion}

We report an unusual presentation of AIP in a young black South African man with a chronic progressive, albeit stuttering, presentation of extensive proximal and distal muscle wasting and weakness developing over the course of 1 year, masquerading as a lower motor neuron syndrome. Our case was atypical in that there were no overt episodes of severe abdominal pain or neuropsychiatric symptoms throughout the course of the illness. A history of recurrent bouts of constipation, probably reflecting the autonomic neuropathy of AIP, was only obtained retrospectively once the diagnosis had been established.

AIP is a dominantly inherited condition resulting from the deficiency of PBG deaminase, which leads to markedly elevated urinary ALA and PBG during an acute attack. Urinary porphyrins are also frequently raised. It is the most common and typically most severe of the acutely presenting porphyrias. ${ }^{[1]}$ The older literature on the neurological manifestations of AIP tends to emphasise the classic triad of abdominal pain, neuropathy and psychosis..$^{[2,3]}$ Autonomic nervous involvement may manifest with tachycardia, fluctuating blood pressure and abdominal pain secondary to ileus. ${ }^{[1}$ The neuropathy usually follows a few days later, with maximum deficit typically reached over 1 month. ${ }^{[1]}$ Characteristically, the neuropathy of AIP is a predominantly motor neuropathy with a predilection for upper limb involvement and proximal more than distal weakness. Unusual sensory symptoms sometimes occur in a bathing trunk distribution. ${ }^{[1]}$

It is held that the neuropathy associated with AIP usually resolves slowly over months and recovery depends on the extent and magnitude of axonal degeneration. ${ }^{[1]}$ Reports have documented gradual improvement of muscle strength over month $s^{[4]}$ to years, but up to $50 \%$ of patients may have residual weakness ${ }^{[5]}$ and $4 \%$ remain quadriparetic. ${ }^{[6]}$ However, fairly rapid improvement on haem arginate, similar to that which our patient experienced, has been observed in several cases. ${ }^{[7.8]}$ The dramatic clinical recovery observed in our patient's proximal muscles over a few days argues for a substantial contribution from neural dysfunction rather than neural degeneration.

The exact mechanism whereby AIP causes neurotoxicity is uncertain, although it has been postulated that ALA (or other metabolites) which are produced in excess by the liver, are neurotoxic. ${ }^{[3,9]}$ This is supported by the benefit seen following liver transplantation in patients with severe AIP. ${ }^{[3]}$ In addition, ALA-induced oxidative damage has been demonstrated in animal models, ${ }^{[10]}$ and ALA levels are also significantly increased in lead poisoning, which may produce a porphyria-like neuropathy. ${ }^{[1]}$ As a critical component of mitochondrial cytochromes, haem participates in the electron transport chain and adenosine triphosphate (ATP) production. Fast axonal transport is highly dependent on this energy. ${ }^{[9]}$ Haem deficiency may result in $\mathrm{Na}^{+}$/ $\mathrm{K}^{+}$pump failure, altered membrane potential with consequent neuronal injury, and even axonal death. ${ }^{[11]}$

Acute attacks of AIP are precipitated by triggers that either directly induce hepatic ALA synthase 1 (ALAS1) activity, or increase the demand for haem synthesis, thereby reducing the negative feedback on ALAS1. ${ }^{[3]}$ These events include menstrual hormonal changes, fasting, smoking, infections, and exposure to porphyrinogenic drugs. Haematin therapy temporarily down-regulates hepatic ALAS1 activity, thereby reducing the overproduction of potentially neurotoxic metabolites by the liver. ${ }^{[1,3]}$ Symptom onset in our patient correlated with his student life, which included erratic meals and alcohol binges. However, relapses following the diagnosis and counselling did not have clear precipitants, although relative undernutrition may have contributed. The absence of clearly defined clinical attacks in our patient complicates future treatment decisions, especially as porphyrin levels and clinical status do not correlate well. ${ }^{[12]}$

\section{Conclusion}

While AIP is known to exist in all racial groups, it remains a poorly diagnosed and documented condition in black Africans, with only a few PBG deaminase gene defects reported. ${ }^{[13]}$ The PBG deaminase $c 445 C>T$ (R149X) mutation in our patient is novel in a black African.

This case illustrates the need to consider AIP as a possible cause of a progressive severe predominantly motor neuropathy, even in the apparent absence of other features associated with hepatic porphyria. 
Acknowledgements. The authors thank Brandon Davidson and Maglona Paul for performing the porphyrin biochemistry and DNA extraction. The PBG deaminase mutation work and porphyrin diagnostic studies were funded in part by the South African MRC and the National Research Foundation (NRF), where PM is the holder of an MRC grant to study the molecular genetics of AIP in South Africa, and has NRF incentive funding as a rated researcher.

1. Albers JW, Fink JK. Porphyric neuropathy. Muscle Nerve 2004;30(4):410-422. [http://dx.doi org/10.1002/mus.20137]

. Becker DM, Kramer S. The neurological manifestations of porphyria: A review. Medicine (Baltimore) 1977;56:411-423. [http://dx.doi.org/10.1097/00005792-197709000-00003]

Puy H, Gouya L, Deybach J. Porphyrias. Lancet 2010;375(9718):924-937. [http://dx.doi.org/10.1016/ S0140-6736(09)61925-5]

4. Kuo HC, Lee MJ, Chuang WL, Huang CC. Acute intermittent porphyria with peripheral neuropathy: A follow-up study after hematin treatment. J Neurol Sci 2007;260(1-2):231-235. [http://dx.doi. org/10.1016/j.jns.2007.03.018

5. Sorensen AWS, With TK. Persistent paresis after porphyric attacks. S Afr Med J 1971;45(Special issue):101-103.
6. Wikberg A, Andersson C, Lithner F. Signs of neuropathy in the lower legs and feet of patients with acute intermittent porphyria. J Intern Med 2000;248(1):27-32. [http://dx.doi.org/10.1046/j.1365-

Diot E, Corcia P, Zannad N, Chauvet MA, Borie MJ, Maillot F. Favorable outcome of acute porphyric neuropathy after treatment with haem arginate. Rev Neurol (Paris) 2007;163(11):1100-1102. [http:// dx.doi.org/10.1016/S0035-3787(07)74184-X

8. Hift RJ, Meissner PN. An analysis of 112 acute porphyric attacks in Cape Town, South Africa: Evidence that acute intermittent porphyria and variegate porphyria differ in susceptibility and severity. Medicine (Baltimore) 2005;84(1):48-60. [http://dx.doi.org/10.1097/01.md.0000152454.56435.f3

9. Lin CS, Lee MJ, Park SB, Kiernan MC. Purple pigments: The pathophysiology of acute porphyric neuropathy. Clin Neurophysiol 2011;122(12):2336-2344. [http://dx.doi.org/10.1016/j. clinph.2011.07.036]

10. Demasi M, Penatti CAA, Delucia R, Bechara EJH. The prooxidant effect of 5-aminolevulinic acid in the brain tissue of rats: Implications in neuropsychiatric manifestations in porphyrias. Free Radic Bio Med 1996;20(3):291-299. [http://dx.doi.org/10.1016/0891-5849(95)02035-7]

11. Lin CS, Krishnan AV, Lee MJ, et al. Nerve function and dysfunction in acute intermittent porphyria. Brain 2008;131(9):2510-2519. [http://dx.doi.org/10.1093/brain/awn152]

12. Gorchein A, Webber R. Delta-aminolevulinic acid in plasma, cerebrospinal fluid, saliva and erythrocytes: Studies in normal, uraemic and porphyric subjects. Clin Sci 1987;72(1):103-112.

13. Robreau-Fraolini AM, Puy H, Aquaron C, et al. Porphobilinogen deaminase gene in African and AfroCarribbean ethnic groups: Mutations causing acute intermittent porphyria and specific intragenic polymorphisms. Hum Genet 2000;107(2):150-159. [http://dx.doi.org/10.1007/s004390000323]

Accepted 26 November 2013 